\title{
ANALYSIS OF ACCELERATION AND NUMERICAL MODELING OF SKELETON SLED MOTION
}

\author{
Martins Irbe, Karlis Agris Gross, Janis Viba, Marina Cerpinska \\ Riga Technical University, Latvia \\ martins.irbe@rtu.lv, kgross@rtu.lv, janis.viba@rtu.lv, marina.cerpinska@rtu.lv
}

\begin{abstract}
The skeleton is a high-speed sport achieving speeds up to $130 \mathrm{~km} \cdot \mathrm{h}^{-1}$ on an ice track, but conditions for faster sliding have not been documented. This paper describes a theoretical model, an experiment and numerical modelling to evaluate the effect of air drag and runner stiffness. A mathematical model was determined for the forward motion of the skeleton down an angled straight ice track that included vertical motion to consider vibrations from a rough ice track. Numerical modelling results were compared with experimental tests performed at a bobsled push-start facility. The skeleton sliding time was logged at the start and end of a $23.7 \mathrm{~m}$ long ice track. The motion was registered by a portable accelerometer attached to the centre of mass on the skeleton base plate. Acceleration down the ice track axis was numerically integrated to calculate the speed and the distance with time. Numerical modelling showed that the speed increased linearly, and the $23.7 \mathrm{~m}$ were too short to see the effect of air drag and runner stiffness on the sliding time. Modelling results showed that despite faster sliding times at conditions of lower air drag, the runner started to vibrate earlier leading to less stable sliding conditions. A higher runner stiffness delayed the onset of vibration. Modelling not only showed conditions that could lead to faster sliding, but also predicted the stability of the skeleton slide, over a longer distance that is available at push-start facilities.
\end{abstract}

Keywords: winter sports, bobsled, skeleton, performance analysis.

\section{Introduction}

Applied research on how objects slide on ice can benefit from collaboration with athletes and trainers, who can share their experience to give feedback from the skeleton ice track. This work will model and analyse the motion of an athlete together with a skeleton sliding down the inclined ice plane. Other researchers have looked at motion of a bobsled and luge on an ice track. The skeleton is the only sport, where it is possible to alter the degree of contact with ice by altering the runner stiffness and this will be investigated here. Stiffness increased by compressing ends when the runners create an arc resulting in less contact with the ice. Beginners prefer a runner setting with a lower stiffness for greater stability. Experienced winter athletes will select a higher stiffness for higher speeds, but this comes at the cost of less control of slider motion on the ice. Athletes presently adjust the stiffness of the runners intuitively. The aim of this research is to develop a mathematical model to predict the motion of the skeleton athlete down an inclined ice plane and to consider the factors, which affect the stiffness variability most.

Motion will be measured with a portable accelerometer and a calibrated timing device at the Bobsled push-start facility to validate a mathematical model and determine the influence of runner stiffness. A simple model with one degree of freedom for a sledbob-skeleton has analysed the effect of different parameters on the time to slide a set distance [1]. A more complicated model with two degrees of freedom is required to define the position of the sled over the 2D track surface [2]. Experimental results from the $23 \mathrm{~m}$ long ice track at the push-start facility will be compared to the results from the mathematical model. The results will be used to look for a dependency of the runner stiffness on the ease of sliding. The mathematical model will be applied for motion along a longer angled ice track than the Bobsled push-start facility, along a $400 \mathrm{~m}$ distance. Consequently, the mathematical model will provide a means to determine the dynamics of motion at greater speeds and evaluate the effect of air resistance on the motion.

Motion analysis has been previously analysed with an accelerometer in situations different from the intended application to be addressed in this work. The start of the skeleton event has been analysed from the moment, at which the athlete commences running until lying down onto the skeleton $[3 ; 4]$. Other cases have considered the motion on the long ice track that involved larger forces encountered at higher speeds [5]. Experiments on the $23 \mathrm{~m}$ long ice track are of secondary importance in this work; the data from the experiments over the short distance are used to verify the mathematical model that it then is further used to model the motion along a $400 \mathrm{~m}$ ice track. Values relating to motion down the ice-track determined by previous researchers will be included in the mathematical model to give the 
best possible prediction. This work will consider a factor not previously reported - the effect of runner stiffness on motion down the ice track.

The mathematical model for motion of the skeleton and the bobsled has involved four factors the friction between the runner and ice, the aerodynamic drag, the mass and the initial velocity. Most attention has been placed on the runner friction with ice and aerodynamic drag. The coefficient of friction has been suggested from calculations related to the rate of bobsled acceleration on an ice track. The mean sled acceleration $a$ has been shown to be proportional to the average speed square divided by the sled mass, $\bar{V}^{2} / m[5]$. Experiments have measured the acceleration with a gyroscope, and the force in the runners from deformation in strain gauges mounted on axles connected to the runners [6]. Friction in the direction of motion has been given to be between 0.02 and 0.08 that changes with speed, but the perpendicular friction has been shown to increase threefold to about 0.3 during turns.

The goal of the research is to minimize the runner friction on ice, account for variations in the sled (skeleton, luge, bobsled) and minimize the aerodynamic drag so as to provide the fastest movement on ice. The objective of this work will be to look at variations in the runner stiffness and the aerodynamic drag that is known to increase with the speed [7]. The aerodynamic drag will be calculated with techniques applied to measure race-car aerodynamics. Drag on a skeleton will be smaller than on a bobsled due to the size, but furthermore influenced by the athlete geometry/size and the position on the skeleton. Computational fluid dynamics have shown that the skeleton is responsible for $9 \%$ of the total drag, but the main contribution is from the athlete. The total drag of the skeleton and the rider is 0.38. Experiments were conducted in a numerical model with Mathcad using numerical values from previously published values.

\section{Mathematical Model of Motion for the Skeleton and Slider}

The mathematical model must identify all forces acting on the object. The model of the skeleton and the slider uses the $X$ axis as the direction of motion (corresponding to the longest side of the sled), the $\mathrm{Y}$ axis as the short side of the sled, and the $\mathrm{Z}$ axis perpendicular to the inclined plane, Fig 1 . The inclined plane has the angle of inclination $\alpha$.

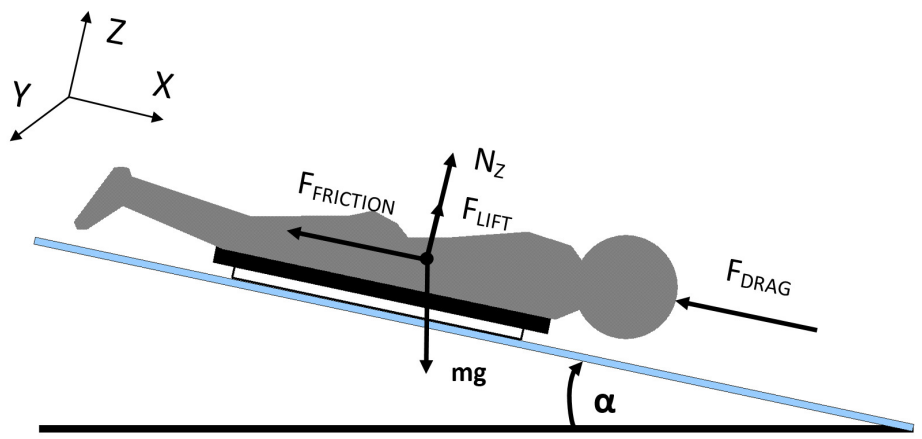

Fig. 1. Forces acting on skeleton from air $\left(\mathrm{F}_{\mathrm{DRAG}}\right.$ and $\left.\mathrm{F}_{\mathrm{LIFT}}\right)$, ice $\left(\mathrm{F}_{\mathrm{FRICTION}}\right)$ and gravity $(\mathrm{mg}$ - system mass and $\mathrm{N}_{\mathrm{Z}}$ - normal reaction)

A simplified mathematical model with one degree of freedom shown as equation (1) has been used by others to describe the motion $[1 ; 5]$.

$$
m \ddot{x}=m \cdot g \cdot \sin (\alpha)-\left(\frac{1}{2} \cdot \rho \cdot C_{D} \cdot A \cdot \dot{x}^{2}-\mu \cdot\left(m \cdot \gamma_{Z}-\frac{1}{2} \cdot \rho \cdot C_{L} \cdot A \cdot \dot{x}^{2}\right)\right),
$$

where $g$-acceleration from to gravity;

$\mu$ - coefficient of friction on ice;

$\rho$ - density of air;

$m$ - mass of the slider and sled;

$A$ - slider and sled frontal area;

$C_{D}$ and $C_{L}$ are aerodynamic drag and aerodynamic lift coefficient;

$\gamma_{y}-$ average vertical acceleration from gravitational and centrifugal accelerations $\left(N_{Z}\right.$ in

Fig. 1.);

$v=\dot{x}-$ longitudinal velocity of skeleton. 
Equation (1) provides motion of the skeleton and driver only along the $\mathrm{x}$ axis. The effect of the runner stiffness on the motion requires an additional equation to account for the spring action parallel to $\mathrm{N}_{\mathrm{Z}}$. The first equation, which describes the motion along the $\mathrm{x}$ axis, is modified to equation (2):

$$
m \ddot{x}=m \cdot g \cdot \sin (\alpha)-\mu \cdot\left[[-c \cdot(z+A \cdot \sin (x))-b \cdot(\dot{z}+A \cdot \cos (x) \cdot \dot{x})] \cdot\left(\frac{1}{2}-\frac{1}{2} \cdot \operatorname{sign}(z+A \cdot \sin (x))\right)\right]-C_{D} \cdot \dot{x}^{2} .
$$

Motion along the $\mathrm{Z}$ axis is given by equation (3):

$$
m \ddot{z}=-m \cdot g \cdot \cos (\alpha)+[-c \cdot(z+A \cdot \sin (x))-b \cdot(\dot{z}+A \cdot \cos (x) \cdot \dot{x})] \cdot\left(\frac{1}{2}-\frac{1}{2} \cdot \operatorname{sign}(z+A \cdot \sin (x))\right),
$$

where $z$-perpendicular to the $\mathrm{x}$ axis displacement;

$A$ - amplitude of the harmonic profile of motion contact surface;

$c, b$ - parameters of runner stiffness and damping;

sign - value +1 or -1 (e.g., sign $=1)$.

Small waves are introduced to create an ice surface that more closely resembles the ice track. The waves are modelled using a harmonic function. As the centre of mass slides along the given track, the sign function simulates small leaps of the skeleton and models a change in the contact forces.

\section{Experiment of the skeleton on the ice track}

An experiment was conducted on the ice track to determine the similarity in the outcome to the numerical model. The experiment was conducted on a straight $23.7 \mathrm{~m}$ long ice track angled at $\alpha=12^{\circ}$. Figure 2 shows the location of the accelerometer fixed to the underside of the skeleton at the centre of mass and the motion of the skeleton together with the slider moving down the ice track at the bobsled push-start facility in Sigulda, Latvia.

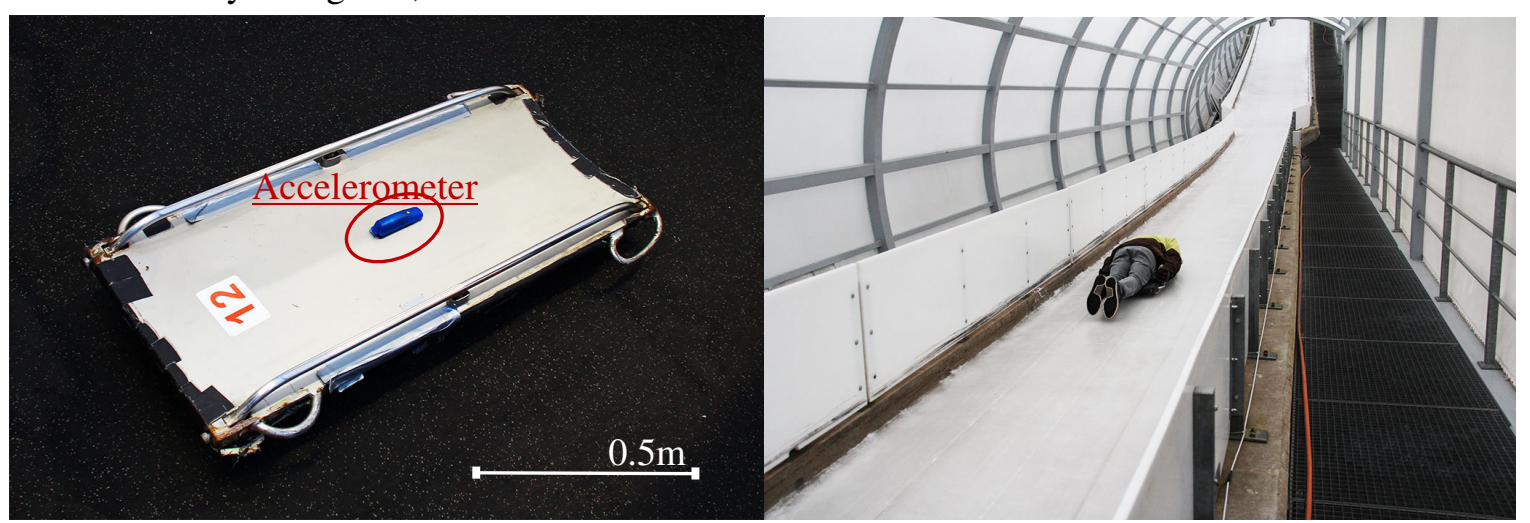

Fig. 2. Skeleton with portable accelerometer and skeleton in motion down the ice track

The sliding time is measured with optical sensors at the top and bottom of the incline after the skeleton starts from sliding from a stationary position. This sliding time was used to calibrate the accelerometer data.

\section{Results and discussion}

Waviness was introduced by a harmonic wave along the x-axis with an amplitude of $1 \mathrm{~cm}$. This resulted in repetitious movement of the skeleton at the same frequency, but the amplitude at least three times higher than the wave in the surface, Fig. 3.

Before conducting an in-depth motion analysis, the outcome from the numerical model was verified with the experimental data. The acceleration given by the accelerometer along the $\mathrm{X}$ axis was integrated to obtain the velocity and integrated a second time to obtain the displacement. The data are shown for 5 seconds, the time taken for the skeleton to slide down the $23.7 \mathrm{~m}$ distance at the skeleton push-start facility. Acceleration shown from the numerical model coincides with the average from the experimental model, Fig 4. The speed from the model compares well with the experimental results, but is slightly lower in the numerical model at the end. The distance data coincide that shows that the portable accelerometer confirmed the general outcome from the numerical model. 


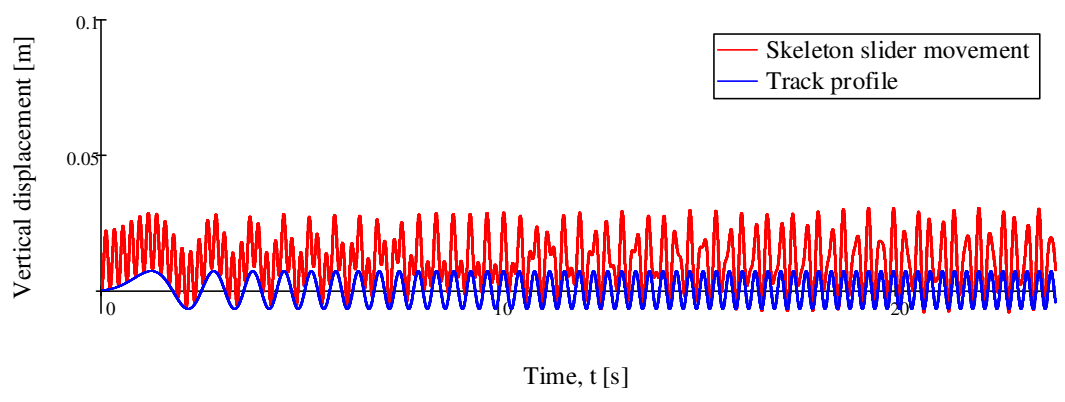

Fig. 3. Harmonic track profile and skeleton-slider centre of mass position in x-direction coinciding with time axis and z-direction shown as height

Air drag, ice friction and skeleton build quality were seen as factors that led to inconsistencies between the numerical data and the experiment data. A slightly higher final speed from the experiment suggests that the input values of air drag and ice friction should be modified for a closer fit. The air drag used in the model was chosen at a smaller value of 0.38 , representative of the lower air drag from the special outfit worn by skeleton sliders [1], compared to the larger air drag from the casual winter attire used by the experimenter. Ice friction also influenced the ability of the numerical model to predict the experimental data. The numerical model confirmed the ice friction between 0.003 and 0.005. The straight acceleration line in the numerical model showed that the harmonic undulations on the surface did not produce accelerations perpendicular to the surface. The experimental acceleration data include contributions from the skeleton as well as the ice track. The noise in the acceleration arose from vibrations in the skeleton base plate as the skeleton moved over the harmonic undulations in the ice surface.

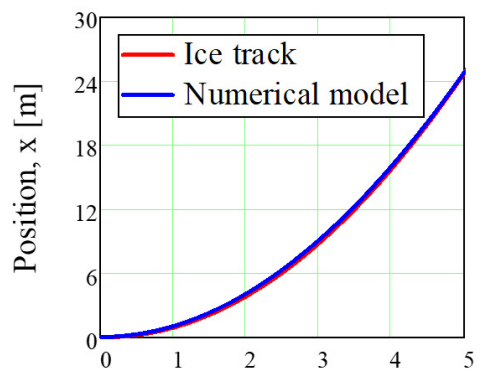

a)

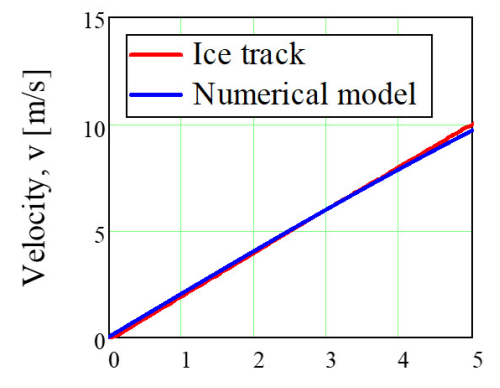

b)

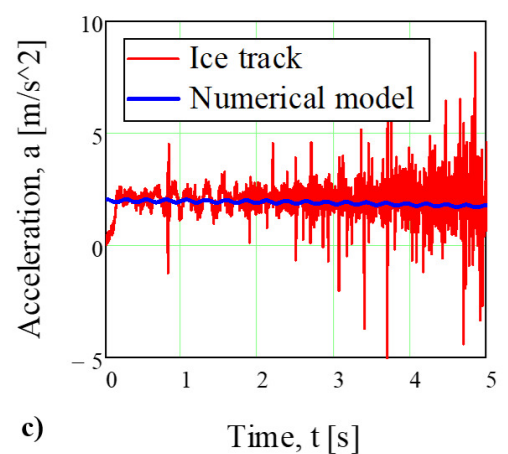

Fig. 4. Comparison of a) displacement, b) velocity and c) acceleration between numerical model and experiment on ice track

The next step after verification of the numerical model was to analyse the effect of the air drag. Two values of the air drag coefficient were used $-C_{D}=0.38$ for a skeleton slider with clothes to lower the air drag in competitions, and $C_{D}=0.44$ for a four men bobsled [7].

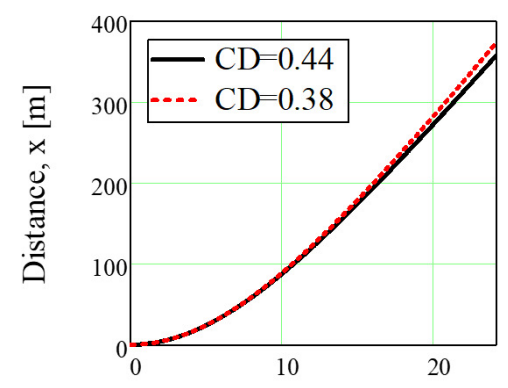

a)

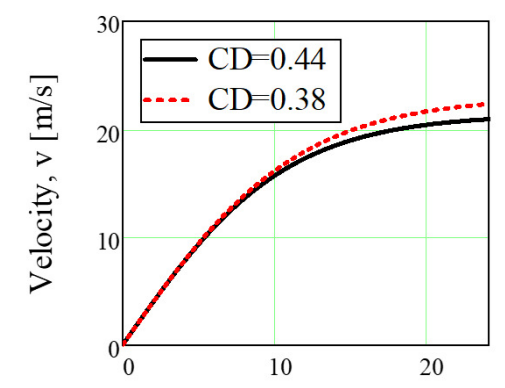

b)

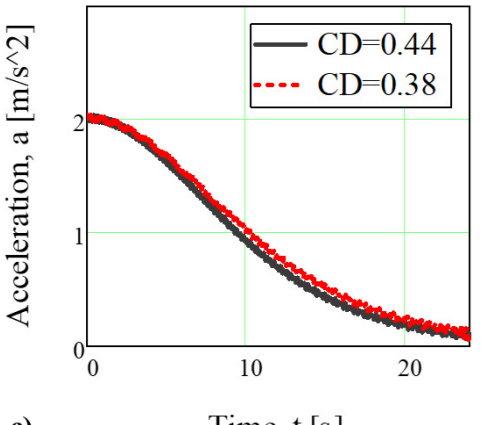

c)

Time, t [s]

Fig. 5. a) position, b) velocity and c) acceleration of skeleton-slider with time: $C_{D}=0.38 C_{D}=0.29$ 
Numerical modelling on a straight ice track showed how the air drag influenced the position, speed and acceleration of the skeleton for a 24 second slide on the ice track with harmonic undulations. The aerodynamic drag exhibited a greater affect at longer times, as suggested by equation (2). The clothing and the skeleton shape thus have a greater influence after 10 seconds of motion, corresponding to a sliding distance of 10 seconds, after which a velocity of $16 \mathrm{~m} \cdot \mathrm{s}^{-1}$ is attained.

The limitations of the short ice track showed the benefit from the numerical model that could be used for a longer sliding distance. Practical experiments at the bobsled push-start facility showed that a short track made it difficult to distinguish the results from each test run. Secondly, the forces change considerably during the initial distance, where a linear increase in velocity was observed. Further the work will consider the numerical model.

Two levels of stiffness were considered in the model to see the effect on motion. The actual values do not correlate with the physical settings on the skeleton. Three levels of air drag were considered to see the collective effect of the runner stiffness and the air drag, Fig 6. With a drag coefficient, $C_{D}$ of 0.29 , the skeleton traversed 399.5 metres in 24 seconds and showed a change in vibration at a higher speed. The amplitude of vibration on the lower stiffness runner began to increase after 14 seconds, corresponding to a distance of $170 \mathrm{~m}$. At a higher stiffness, the amplitude of vibration increased later, at 18 seconds that corresponds to a distance of $250 \mathrm{~m}$.

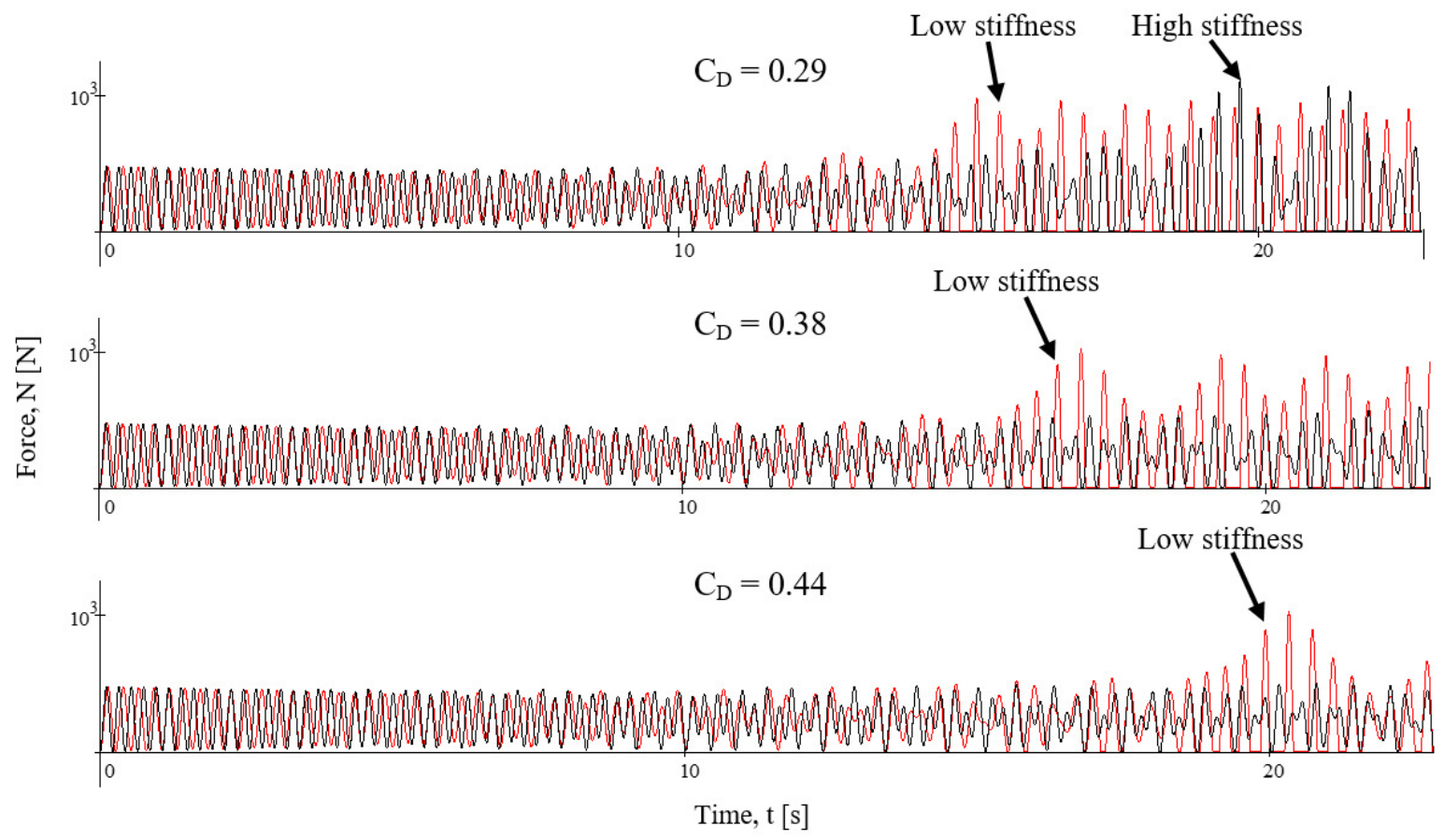

Fig. 6. Normal reaction force from ice track on skeleton runner at different levels of air drag $\left(C_{D}=0.29,0.38\right.$ and 0.44$)$ and two levels of runner stiffness $\left(\right.$ low $-5.7 \times 10^{4}$ and high $\left.-8.7 \times 10^{4}\right)$, showing the onset of greater vibration amplitude

At a higher drag $\left(C_{D}=0.38\right)$, the traversed distance was shorter at $371 \mathrm{~m}$, and the vibrational behaviour was different for the two levels of runner stiffness. At the lower stiffness, the vibration amplitude increased after 16 seconds, at $200 \mathrm{~m}$. The increased in air drag initiated the larger vibration amplitude at a later time. The larger runner stiffness, however, did not show an increase in the vibration amplitude.

For the low stiffness runner, a further increase in the air drag to $C_{D}=0.44$ showed an increase in vibration at still a later time, at $20 \mathrm{~s}$ (a sliding distance of $355 \mathrm{~m}$ ), with the same trend as the intermediate drag setting. The higher stiffness runner did not show any change in the amplitude.

Summing up, a lower air drag increased the speed, but also led to a greater contact pressure resulting from the vibration of the runner on ice, as it slid over the uneven ice surface. A stiffer runner maintained smaller vibration for a longer period of time, indicating that the motion is more stable. 
Numerical modelling showed the influence of air drag and runner stiffness on the speed of sliding and conditions for greater stability. The validation of this model could benefit from a further validation over a longer distance. Assuming the model can predict the motion over a longer distance, then it is possible to determine the optimum condition for a skeleton slider.

Future work will consider the use of strain gauges to confirm the steady and oscillating contact forces in the runner on the ice track.

\section{Conclusions}

1. The numerical model showed that experiments conducted up to 5 seconds are too short to discern differences in the sliding time.

2. Information on the acceleration and time in the direction of sliding was sufficient to determine the coefficient of friction between the skeleton runner and the ice track.

3. Numerical modelling with different values of air drag showed that the contact force frequency and amplitude increased faster at lower air drag conditions.

4. Numerical modelling with different runner stiffness showed that a higher stiffness gives greater stability by delaying the onset of vibration in the runner.

\section{Acknowledgements}

This work has been supported by the ERDF project "The quest for disclosing how surface characteristics affect slideability" (No.1.1.1.1/16/A/129).

The authors gratefully acknowledge the assistance from Dainis Dukurs, Inga Reinina, Janis Lungevics, Ernests Jansons and Liene Pluduma for assistance with experiments at the bobsled pushstart facility.

\section{References}

[1] Roche J., Turnock S., Wright S., An analysis of the interaction between slider physique and descent time for the bob skeleton. Eng. Sports 7(2), 2008, pp. 101-109.

[2] Zhang Y.L., Hubbard M., Huffman R.K., 1995, Optimum control of bobsled steering. J. Optim. Theory Appl 85(1), pp. 1-19.

[3] Bullock N., Martin D., Ross A., Rosemond D., Holland T. and Marino F. Characteristics of the start in women's World Cup skeleton Sport. Biomech 7, 2008, pp. 351-60.

[4] Roberts I. J. M. Skeleton bobsleigh mechanics: athlete-sled interaction. Ph.D. thesis, The University of Edinburgh, 2013.

[5] Braghin F., Cheli F., Melzi S., Sabbioni E. and Maldifassi S., , The engineering approach to winter sports ed F Braghin. Springer, 2016, pp. 183-276.

[6] L. Poirier et al., Experimental analysis of ice friction in the sport of bobsleigh. 2011, Sports Eng., 14(2-4), pp. 67-72.

[7] Lewis O., Aerodynamic analysis of a 2-man bobsleigh. MA thesis, 2006,TU Delft. 